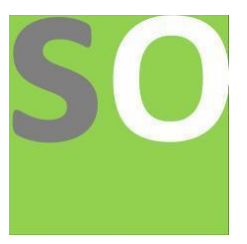

Article title: Maximal prime gaps bounds

Authors: Jan Feliksiak[1]

Affiliations: N. A.[1]

Orcid ids: 0000-0002-9388-1470[1]

Contact e-mail: jan.feliksiak1@yahoo.com

License information: This work has been published open access under Creative Commons Attribution License $\mathrm{http}: / / c r e a t i v e c o m m o n s . o r g / l i c e n s e s / b y / 4.0 /$, which permits unrestricted use, distribution, and reproduction in any medium, provided the original work is properly cited. Conditions, terms of use and publishing policy can be found at https://www.scienceopen.com/.

Preprint statement: This article is a preprint and has not been peer-reviewed, under consideration and submitted to ScienceOpen Preprints for open peer review.

DOI: 10.14293/S2199-1006.1.SOR-.PPWVKRR.v1

Preprint first posted online: 09 February 2021

Keywords: Maximal prime gaps bounds, Ford-Green-Konyagin-Maynard-Tao maximal gaps bound, Maximal prime gaps Infimı Maximal prime gaps Supremum, Maximal prime gaps Lower bound, Maximal prime gaps Upper bound, Eros-Rankin maximal $g$ problem 


\title{
MAXIMAL PRIME GAPS BOUNDS
}

\author{
JAN FELIKSIAK
}

Abstract.

This paper presents research results, pertinent to the maximal prime gaps bounds. Four distinct bounds are presented: Upper bound, Infimum, Supremum and finally the Lower bound. Although the Upper and Lower bounds incur a relatively high estimation error cost, the functions representing them are quite simple. This ensures, that the computation of those bounds will be straightforward and efficient.

The Lower Bound, is essential to address the issue of the value of the lower bound constant $\mathcal{C}$, in the work of Ford et al [16]:

$$
p_{(n+1)}-p_{(n)} \gg \mathcal{C} \frac{(\log N)(\log \log N)(\log \log \log \log N)}{\log \log \log N}
$$

The concluding Corollary in this paper, shows that the value of the constant $\mathcal{C}$ does diverge, although slowly. The constant $\mathcal{C}$, will eventually take any arbitrary value, providing that a large enough $N$ (for $\left.p_{(n+1)} \leq N\right)$ is considered.

The Infimum/Supremum bounds on the other hand are computationally very demanding. Their evaluation entails computations at an extreme level of precision. In return however, we obtain bounds, which provide an extremely close approximation of the maximal prime gaps. The Infimum/Supremum estimation error gradually increases over the range of $p_{n}$, and attains at $p_{n}=$ 18361375334787046697 approximately the value of 0.03 .

\section{(C)2018 Jan Feliksiak}

2000 Mathematics Subject Classification. 0102, 1102, 1103, 11A41, 11K65, 11L20, 11N05.

Key words and phrases. Distribution of primes, Ford-Green-Konyagin-Maynard-Tao maximal gaps problem, maximal prime gaps Lower bound, maximal prime gaps Upper bound, maximal prime gaps Infimum, maximal prime gaps Supremum, Prime Number Theorem, Erdös - Rankin maximal prime gap problem. 


\section{Upper Bound on Maximal Prime Gaps from Historical Perspective}

The topic of the upper bound on the maximal prime gaps, had been discussed in Feliksiak [14] research paper, where the Upper Bound on the Maximal Prime Gaps is shown to be:

Theorem 1.1 (Upper Bound on Maximal Prime Gaps).

The Upper Bound on Maximal Prime Gaps, for all $p_{n} \in \mathbb{N} \mid p_{n} \geq 11$ is given by:

$$
\mathfrak{g}=\left(p_{n+1}-p_{n}\right) \leq 5\left(\log _{10} p_{n}\right)^{2}=\mathcal{U} \mathcal{B}_{\left(p_{n}\right)}
$$

Proof.

For a proof please refer to: pp. 8, Feliksiak [14].

However, already in 1936 a Swedish mathematician Harald Cramér, attempted to formulate the maximal prime gaps upper bound, by implementing a sophisticated heuristic argument. He argued, that there exists at least one prime $p \in \mathbb{N}$, within the interval:

$$
n<p \leq\left(n+(\log p)^{2}\right)
$$

From the Cramér's model we have:

$$
\lim _{n \rightarrow \infty}\left(\frac{p_{(n+1)}-p_{(n)}}{\left(\log p_{(n)}\right)^{2}}\right)=\alpha
$$

H. Cramér asserted that $\alpha=1$. Over the years, the Cramér's Maximal Gaps Conjecture had been considered too strong. In 1995 A. Granville proposed, that there is a bound $\mathrm{M}$ for which:

$$
\lim _{n \rightarrow \infty}\left(\frac{p_{(n+1)}-p_{(n)}}{\left(\log p_{(n)}\right)^{2}}\right)<M
$$

It had been suggested, that $M=2 \mathbf{e}^{-\gamma} \approx 1.12291897$. However, substituting from Theorem 1.1 into the Cramér's model, we obtain:

$$
\alpha=\lim _{n \rightarrow \infty}\left(\frac{p_{(n+1)}-p_{(n)}}{\left(\log p_{(n)}\right)^{2}}\right)=\frac{5}{(\log 10)^{2}} \approx 0.9430585
$$

H. Riesel [25], came up with a test for predictions of the occurrence of maximal gaps of a given size, based on the Cramér's model :

$$
\mathcal{R}=\frac{\log p_{(n)}}{\sqrt{\mathfrak{g}}}
$$

With the limit expected to be:

$$
\lim _{p_{n}, \mathfrak{g} \rightarrow \infty}(\mathcal{R}) \rightarrow 1
$$

From the empirical evidence obtained for the values of $\mathcal{R}$, it seems reasonable: 


\begin{tabular}{lc|c} 
prime & gap size & $\mathcal{R}$ \\
23 & 5 & 1.40224 \\
1129 & 21 & 1.53387 \\
370261 & 111 & 1.21701 \\
4302407359 & 353 & 1.18065 \\
171231342420521 & 805 & 1.15513 \\
218209405436543 & 905 & 1.0975 \\
1425172824437699411 & 1475 & 1.0884
\end{tabular}

However, implementing Theorem 1.1, we determine this limit to be:

$$
\lim _{p_{n}, \mathfrak{g} \rightarrow \infty}(\mathcal{R})=\lim _{p_{n} \rightarrow \infty}\left(\frac{\log p_{(n)}}{\sqrt{5\left(\log _{10} p_{(n)}\right)^{2}}}\right)=\frac{\log 10}{\sqrt{5}} \approx 1.02975
$$

\section{The Maximal Prime Gaps Infimum/Supremum Bounds}

The Infimum/Supremum bounds on the maximal prime gaps, implement Riemann Sums with regular subdivisions of 250000 per unit. This means that over the prime gap, there are $\mathfrak{g} \times 250000$ subdivisions. This was done to overcome technical issues, while evaluating the definite logarithmic integral. All necessary computations were carried out with the aid of Mathematica ${ }^{\circledR}$ software, with numerical precision set to 300000 decimal places.

\section{Maximal Prime Gaps vs Complete Set Of Bounds}

\section{Function Value}

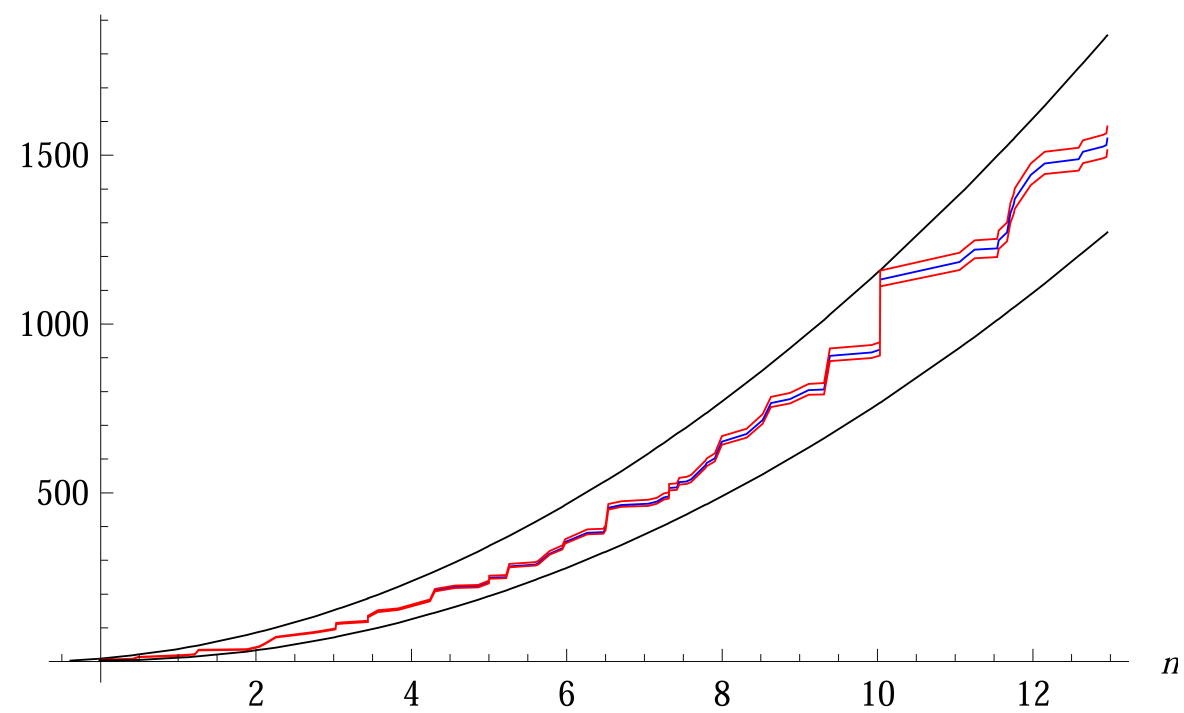

Figure 1. The drawing shows the graph of the Maximal Prime Gaps (Blue), the Infimum/Supremum Bounds (Red) and Lower/Upper Bounds (Black). The figure is drawn w.r.t. $\xi$ (Def. 3.1 ), in the range $p_{n} \in \mathbb{N} \mid 23 \leq p_{n} \leq 18361375334787046697$. 


\section{Remark 2.1.}

The Upper/Lower bounds shown in Figure 1, are given by Theorem 1.1 and Theorem 3.4 respectively. The Auxiliary Infimum/Supremum bounds, presented in the Figure 1, were generated purely for visualization purposes. They are the direct consequence of Theorems 2.6 and 2.7, which specify bounds that are profoundly superior to them. Since for technical reasons, it is impossible to draw the Infimum/Supremum bounds given by Theorems 2.6 and 2.7 vs the Maximal Prime Gaps curve, consequently, only the Infimum/Supremum estimation error graphs are presented. Please refer to Figures 2 and 4. The Auxiliary Infimum/Supremum graphs presented in the Figure 1, can be generated by implementing the Riemann Sum 2.1:

$$
C_{1}\left(\frac{\left(\log _{10} p_{n}\right)^{\alpha}}{(\sqrt{5}-1)^{\beta}}\right)\left[\sum_{k=0}^{(\lim t-1)}\left(\frac{1}{\left(\log \left(x_{1}^{*}+k \Delta x\right)\right)^{\beta}}\right) \Delta x\right]+C_{2}
$$

With the constants (Auxiliary Infimum) defined by:

$$
C_{1}=\frac{959758 \pi}{1000000}, \alpha=1, \beta=1.020 \text { and } C_{2}=(-1)
$$

With the constants (Auxiliary Supremum) defined by:

$$
C_{1}=\frac{9359 \pi}{10000}, \alpha=1, \beta=1.00250 \text { and } C_{2}=0.05
$$

and implementing Definitions 2.1 through 2.5. The estimation error at the last maximal prime gap at $p_{n}=18361375334787046697$, in the case of the Auxiliary Infimum is $\approx 35.328$ (with a global min. at $p_{n}=1327 \approx 0.58286$ ), while in the case of the Auxiliary Supremum it is $\approx 35.3281$. Both bounds are defined from $p_{n}=23$.

First, we make a few general definitions valid within the entire section. In this section, the Riemann sums are implemented, with regular partitioning of the maximal prime gap interval. The number of regular divisions, between any two consecutive integers equals 250000. Maximal Prime Gap of a size $\mathfrak{g}$, is a gap that strictly exceeds in size, any prime gap that precedes it. Let $p_{i}$ and $p_{c}$ denote the initial prime at which the Maximal Prime Gap begins and the concluding prime at which the Maximal Prime Gap ends, respectively.

Definition 2.1 (Maximal Prime Gap).

$$
\mathfrak{g}=p_{c}-p_{i}
$$

Definition 2.2 (Number of divisions of a Regular Partition).

$$
d=250000
$$

Definition 2.3 (Summation Limit).

$$
\text { limt }=d \times \mathfrak{g}
$$

\section{Definition 2.4.}

$$
\Delta x=\frac{\mathfrak{g}}{\lim t}=\frac{1}{d}
$$

Definition 2.5 (Initial Element of Summation).

$$
x_{1}^{*}=p_{i}+\left(\frac{1}{2 d}\right)
$$




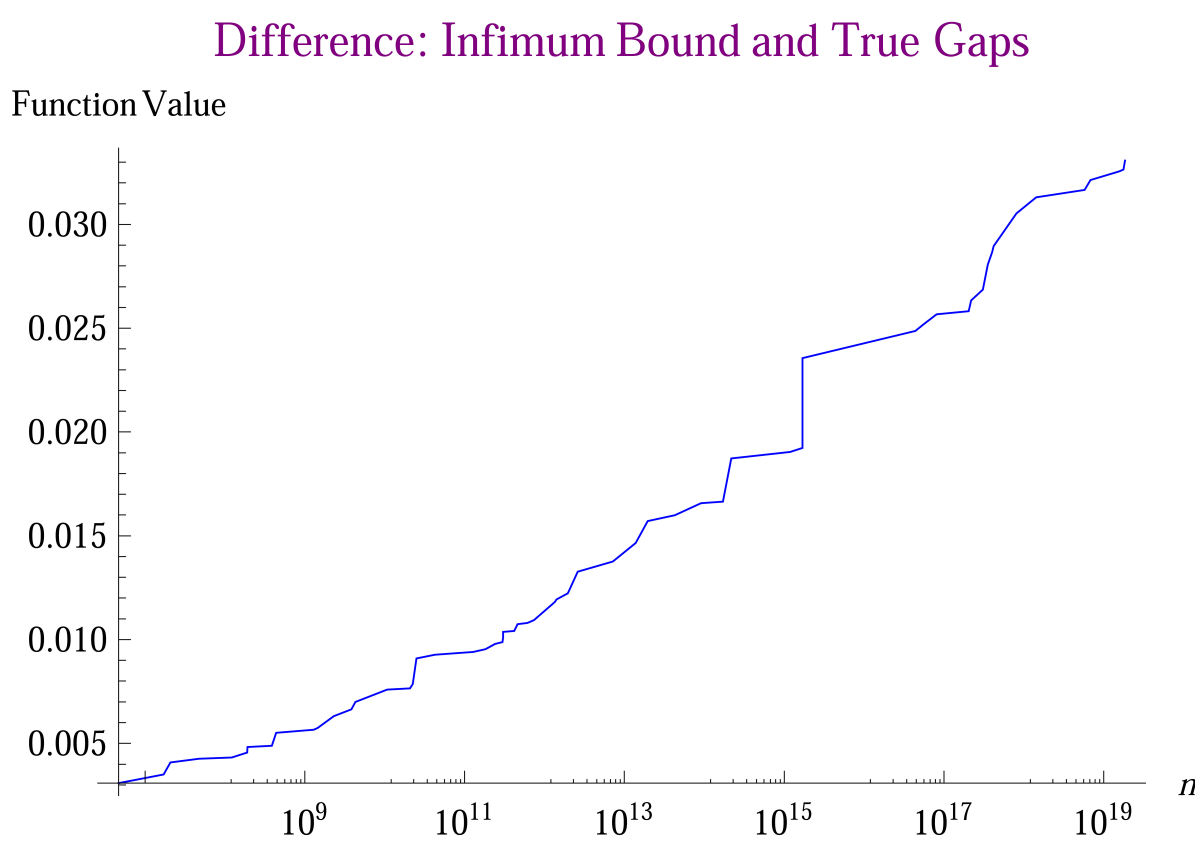

Figure 2. The drawing shows the graph of the estimation error made by the Infimum Bound. The figure is drawn w.r.t. $p_{n}$, in the range $p_{n} \in \mathbb{N} \mid 4652353 \leq p_{n} \leq 18361375334787046697$.

Theorem 2.6 (Maximal Prime Gaps Infimum).

The Infimum Bound on the Maximal Prime Gaps, for $p_{i} \in \mathbb{N} \mid p_{i} \geq 23$ is given by:

$$
\mathcal{I N}_{\mathcal{F}_{p_{i}}}=C_{1}\left(\frac{\left(\log _{10} p_{i}\right)^{\alpha}}{(\sqrt{5}-1)^{\beta}}\right)\left[\sum_{k=0}^{\left({ }^{l i m t-1)}\right.}\left(\frac{1}{\left(\log \left(x_{1}^{*}+k \Delta x\right)\right)^{\beta}}\right) \Delta x\right]
$$

With the constants:

$$
C_{1}=\frac{907727 \pi}{1000000}, \alpha=1.00187651 \text { and } \beta=1.00187877
$$

Proof.

The Riemann sum 2.2 approximates the logarithmic integral over the maximal prime gaps:

$$
\begin{aligned}
C_{1}\left(\frac{\left(\log _{10} p_{i}\right)^{\alpha}}{(\sqrt{5}-1)^{\beta}}\right) & \int_{p_{i}}^{p_{c}}\left(\frac{1}{(\log t)^{\beta}}\right) d t \\
& \lesssim C_{1}\left(\frac{\left(\log _{10} p_{i}\right)^{\alpha}}{(\sqrt{5}-1)^{\beta}}\right)\left[\sum_{k=0}^{(\lim t-1)}\left(\frac{1}{\left(\log \left(x_{1}^{*}+k \Delta x\right)\right)^{\beta}}\right) \Delta x\right]
\end{aligned}
$$

Let's denote a maximal prime gap:

$$
\mathcal{G}_{N}=\max _{p_{c} \leq N}\left(p_{c}-p_{i}\right)
$$


In 1931 Westzynthius [31] proved,

$$
\lim _{p_{c} \rightarrow \infty}\left(\frac{\mathcal{G}_{N}}{\log p_{c}}\right) \rightarrow \infty
$$

Equivalently, in the case of the maximal prime gaps:

$$
\lim _{p_{c} \rightarrow \infty}\left(\frac{p_{c}-p_{i}}{\log p_{c}}\right)=\lim _{p_{c} \rightarrow \infty}\left(\frac{p_{c}-p_{i}}{\left(\log p_{c}\right)^{\beta}}\right) \rightarrow \infty
$$

By the PNT for all $p_{i} \in \mathbb{N} \mid p_{i} \geq 23$ we may estimate the logarithmic integral, hence the Riemann's Sum:

$$
\frac{p_{c}-p_{i}}{\left(\log p_{c}\right)^{\beta}} \lesssim \int_{p_{i}}^{p_{c}} \frac{1}{(\log t)^{\beta}} d t \lesssim \sum_{k=0}^{(\text {limt }-1)}\left(\frac{1}{\left(\log \left(x_{1}^{*}+k \Delta x\right)\right)^{\beta}}\right) \Delta x
$$

Clearly,

$$
\lim _{p_{i} \rightarrow \infty} C_{1}\left(\frac{\left(\log _{10} p_{i}\right)^{\alpha}}{(\sqrt{5}-1)^{\beta}}\right) \rightarrow \infty
$$

Consequently, we have:

$$
\begin{aligned}
& \lim _{p_{i} \rightarrow \infty}\left(C_{1}\left(\frac{\left(\log _{10} p_{i}\right)^{\alpha}}{(\sqrt{5}-1)^{\beta}}\right) \int_{p_{i}}^{p_{c}}\left(\frac{1}{(\log t)^{\beta}}\right) d t\right) \\
& =\lim _{p_{i} \rightarrow \infty}\left(C_{1}\left(\frac{\left(\log _{10} p_{i}\right)^{\alpha}}{(\sqrt{5}-1)^{\beta}}\right)\left[\sum_{k=0}^{(\lim t-1)}\left(\frac{1}{\left(\log \left(x_{1}^{*}+k \Delta x\right)\right)^{\beta}}\right) \Delta x\right]\right) \rightarrow \infty
\end{aligned}
$$

Suppose that Theorem 2.6 is false for some $p_{i} \in \mathbb{N} \mid p_{i} \geq 4652353$. Define now:

$$
\mathcal{M P}_{p_{p_{i}}}=\left(\mathfrak{g}-\frac{\log \log \log p_{i}}{330}\right) \quad \forall p_{i} \in \mathbb{N} \mid p_{i} \geq 4652353
$$

The natural $\operatorname{logarithm}$ function $\left(\log \log \log p_{i}\right) / 330$ for $p_{i} \geq 4652353$, is a positive, increasing function, which at $p_{i}=4652353$ attains approx. 0.00304478 and diverges. Thus, in accordance with the hypothesis, at the maximal gaps we must have:

$$
\mathfrak{g}-C_{1}\left(\frac{\left(\log _{10} p_{i}\right)^{\alpha}}{(\sqrt{5}-1)^{\beta}}\right)\left[\sum_{k=0}^{(\text {limt }-1)}\left(\frac{1}{\left(\log \left(x_{1}^{*}+k \Delta x\right)\right)^{\beta}}\right) \Delta x\right]<0
$$

Since $\mathcal{M P G}_{p_{i}}<\mathfrak{g} \quad \forall p_{i} \geq 4652353$, necessarily therefore:

$$
\begin{aligned}
& \mathcal{M P} \mathcal{G}_{p_{i}}-C_{1}\left(\frac{\left(\log _{10} p_{i}\right)^{\alpha}}{(\sqrt{5}-1)^{\beta}}\right)\left[\sum_{k=0}^{\left({ }^{\text {limt }}-1\right)}\left(\frac{1}{\left(\log \left(x_{1}^{*}+k \Delta x\right)\right)^{\beta}}\right) \Delta x\right] \\
& =\left(\mathfrak{g}-\mathcal{I N F}_{p_{i}}\right)-\frac{\log \log \log p_{i}}{330}=\text { (est. error) }-\frac{\log \log \log p_{i}}{330}<0
\end{aligned}
$$


Thus necessarily, the ratio $a_{j}$ must be:

$$
\begin{aligned}
a_{j}= & \frac{\text { estimation error }}{\left(\log \log \log p_{i}\right) / 330} \\
& =\frac{\left(\mathfrak{g}-C_{1}\left(\frac{\left(\log _{10} p_{i}\right)^{\alpha}}{(\sqrt{5}-1)^{\beta}}\right)\left[\sum_{k=0}^{(\lim t-1)}\left(\frac{1}{\left(\log \left(x_{1}^{*}+k \Delta x\right)\right)^{\beta}}\right) \Delta x\right]\right)}{\left(\left(\log \log \log p_{i}\right) / 330\right)}<1
\end{aligned}
$$

where $j$ is the Maximal Gap ordinal number. The ratio at $j=20, p_{i}=4652353$, attains approx. 1.01267 and strictly increases at every step $j$, diverging as $j$ increases. Please refer to Figure 3. Consequently this implies, that we have a contradiction to the initial assumption. This implies that:

$$
\lim _{p_{i} \rightarrow \infty}\left(\mathfrak{g}-C_{1}\left(\frac{\left(\log _{10} p_{i}\right)^{\alpha}}{(\sqrt{5}-1)^{\beta}}\right)\left[\sum_{k=0}^{(\lim t-1)}\left(\frac{1}{\left(\log \left(x_{1}^{*}+k \Delta x\right)\right)^{\beta}}\right) \Delta x\right]\right) \rightarrow \infty
$$

which in turn implies, that Theorem 2.6 holds for $p_{i} \in \mathbb{N} \mid p_{i} \geq 4652353$. For $23 \leq p_{i} \leq 4652353$, direct computer evaluation demonstrates that, Theorem 2.6 holds in this range as well. Please refer to Table 2. Hence, Theorem 2.6, holds as stated for all $p_{i} \in \mathbb{N} \mid p_{i} \geq 23$. Concluding the proof.

$$
\text { Est. Error } /\left(\frac{\log \left[\log \left[\log \left[p_{n}\right]\right]\right]}{330}\right)
$$

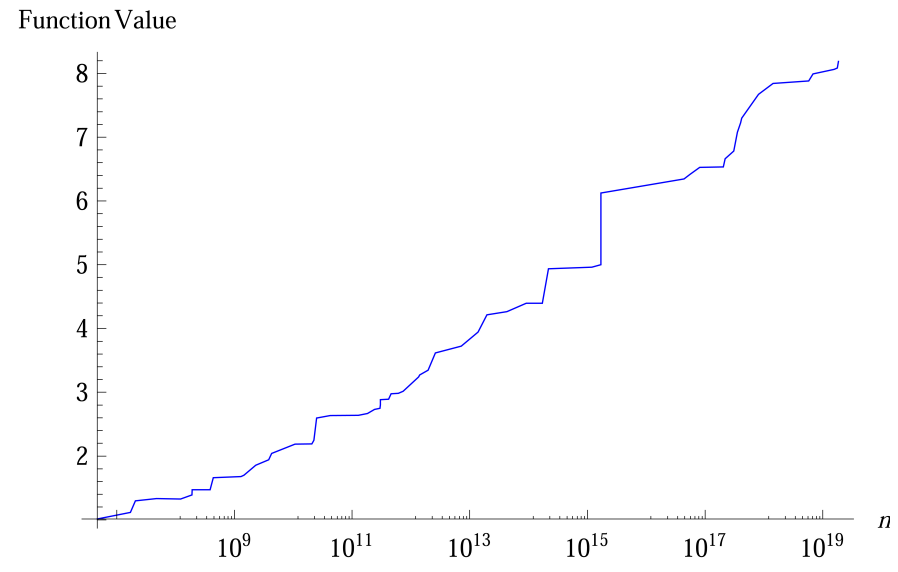

Figure 3. The drawing shows ratio 2.12. The log-linear figure is drawn w.r.t. $p_{i} \in \mathbb{N}$ s.t. $4652353 \leq p_{i} \leq 18361375334787046697$.

Theorem 2.7 (Maximal Prime Gaps Supremum).

The Supremum Bound on the Maximal Prime Gaps, for $p_{n} \in \mathbb{N} \mid p_{n} \geq 155921$ is given by:

$$
C_{1}\left(\frac{\left(\log _{10} p_{n}\right)^{\alpha}}{(\sqrt{5}-1)^{\beta}}\right)\left[\sum_{k=0}^{(\text {limt }-1)}\left(\frac{1}{\left(\log \left(x_{1}^{*}+k \Delta x\right)\right)^{\beta}}\right) \Delta x\right]
$$




\section{Difference: Supremum Bound and True Gaps}

Function Value

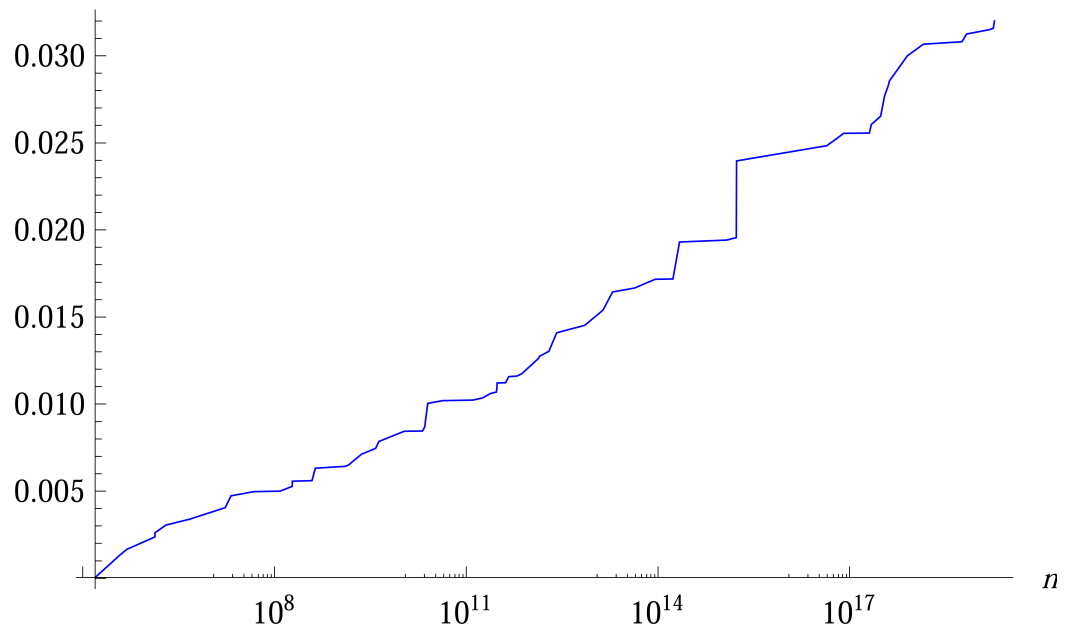

Figure 4. This log-linear drawing shows the graph of the estimation error made by the Supremum Bound. Please refer to Table 3 in the Appendix. The figure is drawn w.r.t. $p_{n} \in \mathbb{N}$, in the range $155921 \leq p_{n} \leq 18361375334787046697$.

With the constants:

$$
C_{1}=\frac{907765 \pi}{1000000}, \alpha=1.00187651 \text { and } \beta=1.00187874
$$

Remark 2.2. The range of the Supremum Bound may be extended, to include all primes $p_{n} \geq 523$ by adding the constant 0.0592 and re-computing pertinent data.

Proof.

The Riemann sum 2.14 approximates the logarithmic integral over the maximal prime gaps:

$$
\begin{aligned}
C_{1}\left(\frac{\left(\log _{10} p_{i}\right)^{\alpha}}{(\sqrt{5}-1)^{\beta}}\right) \int_{p_{i}}^{p_{c}}\left(\frac{1}{(\log t)^{\beta}}\right) d t \\
\quad \lesssim C_{1}\left(\frac{\left(\log _{10} p_{i}\right)^{\alpha}}{(\sqrt{5}-1)^{\beta}}\right)\left[\sum_{k=0}^{(\text {limt }-1)}\left(\frac{1}{\left(\log \left(x_{1}^{*}+k \Delta x\right)\right)^{\beta}}\right) \Delta x\right]
\end{aligned}
$$

Let's denote a maximal prime gap:

$$
\mathcal{G}_{N}=\max _{p_{c} \leq N}\left(p_{c}-p_{i}\right)
$$

In 1931 Westzynthius [31] proved,

$$
\lim _{p_{c} \rightarrow \infty}\left(\frac{\mathcal{G}_{N}}{\log p_{c}}\right) \rightarrow \infty
$$


Equivalently, in the case of the maximal prime gaps:

$$
\lim _{p_{c} \rightarrow \infty}\left(\frac{p_{c}-p_{i}}{\log p_{c}}\right)=\lim _{p_{c} \rightarrow \infty}\left(\frac{p_{c}-p_{i}}{\left(\log p_{c}\right)^{\beta}}\right) \rightarrow \infty
$$

By the PNT for all $p_{i} \in \mathbb{N} \mid p_{i} \geq 155921$ we may estimate the logarithmic integral, hence the Riemann's Sum:

$$
\frac{p_{c}-p_{i}}{\left(\log p_{c}\right)^{\beta}} \lesssim\left[\int_{p_{i}}^{p_{c}} \frac{1}{(\log t)^{\beta}} d t\right] \lesssim\left[\sum_{k=0}^{(\lim t-1)}\left(\frac{1}{\left(\log \left(x_{1}^{*}+k \Delta x\right)\right)^{\beta}}\right) \Delta x\right]
$$

Clearly,

$$
\lim _{p_{i} \rightarrow \infty} C_{1}\left(\frac{\left(\log _{10} p_{i}\right)^{\alpha}}{(\sqrt{5}-1)^{\beta}}\right) \rightarrow \infty
$$

Consequently, we have:

$$
\begin{aligned}
& \lim _{p_{i} \rightarrow \infty}\left(C_{1}\left(\frac{\left(\log _{10} p_{i}\right)^{\alpha}}{(\sqrt{5}-1)^{\beta}}\right) \int_{p_{i}}^{p_{c}}\left(\frac{1}{(\log t)^{\beta}}\right) d t\right) \\
= & \lim _{p_{i} \rightarrow \infty}\left(C_{1}\left(\frac{\left(\log _{10} p_{i}\right)^{\alpha}}{(\sqrt{5}-1)^{\beta}}\right)\left[\sum_{k=0}^{(\operatorname{limt}-1)}\left(\frac{1}{\left(\log \left(x_{1}^{*}+k \Delta x\right)\right)^{\beta}}\right) \Delta x\right]\right) \rightarrow \infty
\end{aligned}
$$

Suppose that Theorem 2.7 is false for some $p_{i} \in \mathbb{N} \mid p_{i} \geq 1357201$. Define now:

$$
\mathcal{M P}_{p_{i}}=\left(\mathfrak{g}+\frac{\log \log \log p_{i}}{380}\right) \quad \forall p_{i} \in \mathbb{N} \mid p_{i} \geq 1357201
$$

The natural $\operatorname{logarithm}$ function $\left(\log \log \log p_{i}\right) / 380$ for $p_{i} \geq 1357201$, is a positive, increasing function, which at $p_{i}=1357201$ attains approx. 0.0025623 and diverges. Thus, in accordance with the hypothesis, at the maximal gaps we must have:

$$
C_{1}\left(\frac{\left(\log _{10} p_{i}\right)^{\alpha}}{(\sqrt{5}-1)^{\beta}}\right)\left[\sum_{k=0}^{(\text {limt-1) }}\left(\frac{1}{\left(\log \left(x_{1}^{*}+k \Delta x\right)\right)^{\beta}}\right) \Delta x\right]-\mathfrak{g}<0
$$

Since $\mathcal{M P \mathcal { G }} \mathcal{G}_{p_{i}}>\mathfrak{g} \quad \forall p_{i} \in \mathbb{N} \mid p_{i} \geq 1357201$, necessarily therefore:

$$
C_{1}\left(\frac{\left(\log _{10} p_{i}\right)^{\alpha}}{(\sqrt{5}-1)^{\beta}}\right)\left[\sum_{k=0}^{(\text {limt-1) }}\left(\frac{1}{\left(\log \left(x_{1}^{*}+k \Delta x\right)\right)^{\beta}}\right) \Delta x\right]-\mathcal{M P \mathcal { G }}_{p_{i}}
$$

$$
=(\mathcal{S U P}-\mathfrak{g})-\left(\log \log \log p_{i}\right) / 380=(\text { estimation error })-\left(\log \log \log p_{i}\right) / 380<0
$$

Thus necessarily, the ratio must be:

$$
\begin{aligned}
a_{j}= & \frac{(\text { estimation error })}{\left(\log \log \log p_{i}\right) / 380} \\
& =\frac{\left(C_{1}\left(\frac{\left(\log _{10} p_{i}\right)^{\alpha}}{(\sqrt{5}-1)^{\beta}}\right)\left[\sum_{k=0}^{(\lim t-1)}\left(\frac{1}{\left(\log \left(x_{1}^{*}+k \Delta x\right)\right)^{\beta}}\right) \Delta x\right]-\mathfrak{g}\right)}{\left(\left(\log \log \log p_{i}\right) / 380\right)}<1
\end{aligned}
$$

where $j$ is the Maximal Gap ordinal number. The ratio at $j=18, p_{i}=1357201$ attains approx. 1.01717452 and strictly increases at every step $j$, diverging as $j$ 
increases. Please refer to Figure 5. Consequently this implies, that we have a contradiction to the initial assumption. This implies that:

$$
\lim _{p_{i} \rightarrow \infty}\left(C_{1}\left(\frac{\left(\log _{10} p_{i}\right)^{\alpha}}{(\sqrt{5}-1)^{\beta}}\right)\left[\sum_{k=0}^{(\lim t-1)}\left(\frac{1}{\left(\log \left(x_{1}^{*}+k \Delta x\right)\right)^{\beta}}\right) \Delta x\right]-\mathfrak{g}\right) \rightarrow \infty
$$

which in turn implies that Theorem 2.7 holds for $p_{i} \in \mathbb{N} \mid p_{i} \geq 1357201$. For $155921 \leq p_{i} \leq 1357201$ direct computer evaluation demonstrates that, Theorem 2.7 holds in this range as well. Please refer to Table 3. Hence, Theorem 2.7 holds as stated for all $p_{i} \in \mathbb{N} \mid p_{i} \geq 155921$. Concluding the proof.

$$
\text { Est. Error /( } \left.\frac{\log \left[\log \left[\log \left[p_{n}\right]\right]\right]}{380}\right)
$$

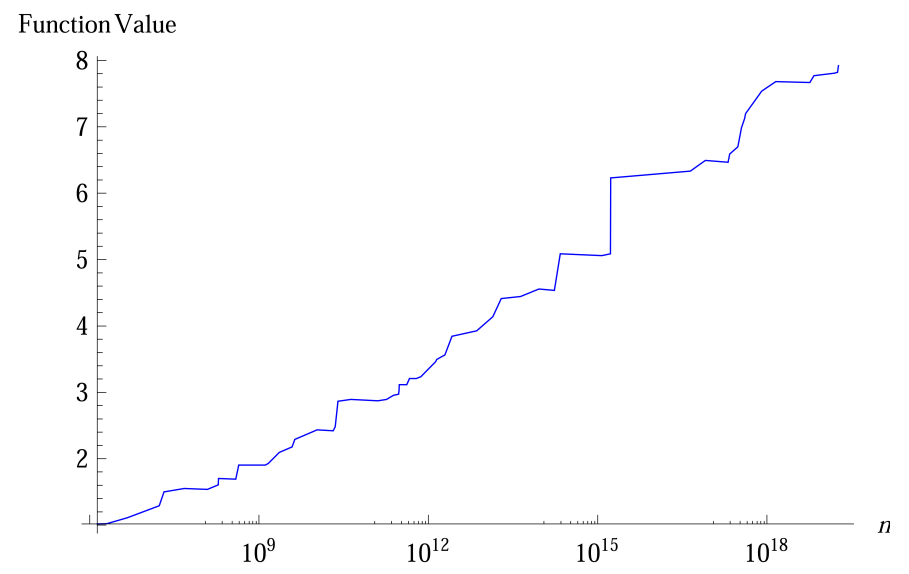

Figure 5. The drawing shows ratio 2.23. The figure is drawn w.r.t. $p_{i} \in \mathbb{N}$, s.t. $1357201 \leq p_{n} \leq 18361375334787046697$.

\section{Lower Bound on Maximal Prime Gaps with Historical Background}

From the Prime Number Theorem we have that an average gap between consecutive primes is given by $\log n$ for any $n \in \mathbb{N}$. There exist however prime gaps much shorter - containing only a single composite number, and gaps which are much longer than average - the maximal prime gaps. In 1929 R. Backlund [1] published a paper in which he proved the lower bound on the maximal prime gaps:

$$
\mathfrak{g}=p_{(n+1)}-p_{(n)}>(2-\epsilon) \log p_{(n)} \text { for any } \epsilon>0
$$

This was the first notable result in this area. In 1931 Westzynthius [31] proved the normalized prime gap limit superior:

$$
\limsup _{p_{(n+1)} \rightarrow \infty}\left(\frac{\mathfrak{g}}{\log p_{(n+1)}}\right)=\limsup _{p_{(n+1)} \rightarrow \infty}\left(\frac{p_{(n+1)}-p_{(n)}}{\log p_{(n+1)}}\right) \rightarrow \infty
$$


The result 3.1 had been improved upon in 1935 by Paul Erdös [10] who proved that for $c>0$ we have:

$$
\mathfrak{g}=p_{(n+1)}-p_{(n)}>\frac{c(\log N)(\log \log N)}{(\log \log \log N)^{2}}
$$

Robert Rankin [24] in 1938 proved that for $c>0$ we have:

$$
\mathfrak{g}=p_{(n+1)}-p_{(n)}>\frac{c(\log N)(\log \log N)(\log \log \log \log N)}{(\log \log \log N)^{2}}
$$

In a more recent development, Kevin Ford Et Al. [16] in 2016 established for all $p_{(n+1)} \in \mathbb{N} \mid p_{(n+1)} \leq N$, a bound of the form:

$$
\mathfrak{g}=p_{(n+1)}-p_{(n)} \gg \frac{(\log N)(\log \log N)(\log \log \log \log N)}{\log \log \log N}
$$

with the constant $c$ being implicit. For clarity of presentation, some graphs implement a variant of a logarithmic scaling of the horizontal axis, given by:

Definition 3.1 (Scaling factor). $\xi=\frac{\log _{10}\left(\frac{p(n)}{24}\right)}{\log _{10}(24)}$

Definition 3.2 (Alpha Constant). $\alpha=\left(\frac{\left(5+(\log 10)^{2}\right)}{(\log 10)^{2}}\right)$

Definition 3.3 (Beta Constant). $\beta=\left(\frac{(\exp 1) \pi}{2}\right)$

Theorem 3.4 (Maximal Prime Gaps Lower Bound).

The maximal prime gaps Lower Bound, for all $p_{(n)} \in \mathbb{N} \mid p_{(n)} \geq 23$ is given by:

$$
\mathfrak{g}=p_{(n+1)}-p_{(n)} \geq \mathcal{L} \mathcal{B}_{p_{n}}=\mathcal{M} \times\left(5\left(\log _{10} p_{n}\right)^{\alpha}-7\left(\log _{10} p_{n}\right)^{(2 \gamma)}\right)+\beta
$$

Where $\alpha$ and $\beta$ are given by the Definitions 3.2 and 3.3 respectively, $\gamma$ is the EulerMascheroni constant and $\mathcal{M}$ is given by:

$$
\mathcal{M}=\left(1-\frac{\sqrt{5}-1}{\log _{10} p_{n}}\right) \text {. }
$$

Proof.

Theorem 1.1 gives for all $p_{n} \in \mathbb{N} \mid p_{n} \geq 11$, the upper bound on the maximal prime gaps:

$$
\mathfrak{g}=\left(p_{n+1}-p_{n}\right) \leq 5\left(\log _{10} p_{n}\right)^{2}
$$

Clearly, for all $p_{n} \in \mathbb{N} \mid p_{n} \geq 23$ :

$$
\mathcal{L B}_{p_{n}}=\mathcal{M} \times\left(5\left(\log _{10} p_{n}\right)^{\alpha}-7\left(\log _{10} p_{n}\right)^{(2 \gamma)}\right)+\beta<5\left(\log _{10} p_{n}\right)^{2}
$$

Suppose that Theorem 3.4 is false, for $p_{n} \in \mathbb{N} \mid p_{n} \geq 155921$. Consequently,

$$
\mathcal{L B}_{p_{n}}=\mathcal{M} \times\left(5\left(\log _{10} p_{n}\right)^{\alpha}-7\left(\log _{10} p_{n}\right)^{(2 \gamma)}\right)+\beta>\left(p_{n+1}-p_{n}\right)=\mathfrak{g}
$$

This implies that:

$$
\frac{p_{n}}{\mathcal{M} \times\left(5\left(\log _{10} p_{n}\right)^{\alpha}-7\left(\log _{10} p_{n}\right)^{(2 \gamma)}\right)+\beta}-\frac{p_{n}}{\left(p_{n+1}-p_{n}\right)}<0
$$


Cauchy Root Test at $j=13, p_{n}=155921$ :

$$
\sqrt[j]{\left|a_{j}\right|}=\sqrt[j]{\left|\left(\frac{p_{n}}{\mathcal{M} \times\left(5\left(\log _{10} p_{n}\right)^{\alpha}-7\left(\log _{10} p_{n}\right)^{(2 \gamma)}\right)+\beta}\right)-\left(\frac{p_{n}}{\mathfrak{g}}\right)\right|} \approx 1.72585
$$

Where $j$ is the Ordinal Number of the maximal gap and $p_{n}$ is the prime at which the maximal gap commences. Furthermore, the Cauchy's Root Test at every step $j$ exceeds 1, while decreasing strictly from above towards 1 . Please refer to Figure 6 . Hence, by the definition of the Root Test, the sequence $\left|a_{j}\right|$ diverges. In accordance with the initial hypothesis, this implies that:

$$
\left(\frac{p_{n}}{\mathcal{M} \times\left(5\left(\log _{10} p_{n}\right)^{\alpha}-7\left(\log _{10} p_{n}\right)^{(2 \gamma)}\right)+\beta}-\frac{p_{n}}{\left(p_{n+1}-p_{n}\right)}\right) \rightarrow-\infty
$$

However, at $p_{n}=155921$ the difference 3.13 attains approx. 698.282 and rapidly diverges. Please refer to Figure 6. Hence, we have a contradiction to the initial assumption, necessarily this implies that for any $p_{n} \geq 155921$ :

$$
\mathcal{M} \times\left(5\left(\log _{10} p_{n}\right)^{\alpha}-7\left(\log _{10} p_{n}\right)^{(2 \gamma)}\right)+\beta<\mathfrak{g}
$$

Hence, Theorem 3.4 holds for $p_{n} \in \mathbb{N} \mid p_{n} \geq 155921$. For $23 \leq p_{n} \leq 155921$, direct computer evaluation demonstrates, that Theorem 3.4 holds in this range as well. Please refer to Table 4. This implies that Theorem 3.4 holds as stated for all $p_{n} \in \mathbb{N} \mid p_{n} \geq 23$. Concluding the proof.
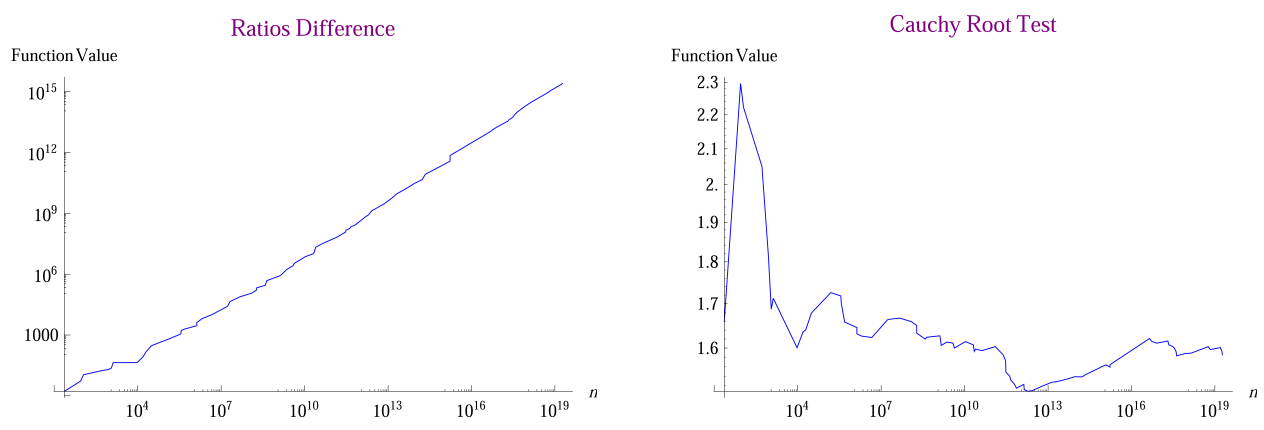

Figure 6 . The L.H. log-log drawing shows the graph of the difference of ratios 3.11. The R.H. log-linear drawing shows the graph of the Cauchy Root Test 3.12. The figures are drawn w.r.t. $p_{n} \in \mathbb{N}$, in the range $23 \leq p_{n} \leq 18361375334787046697$. 

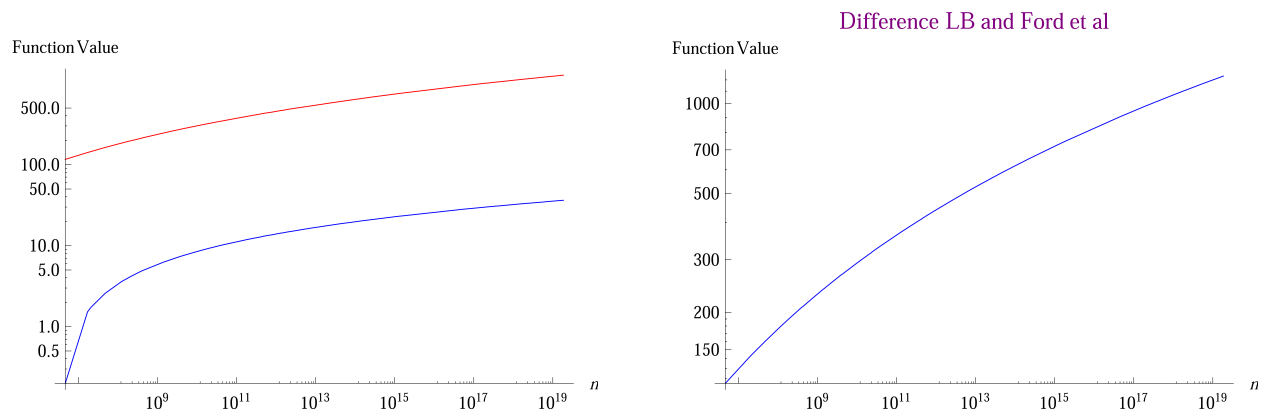

Figure 7. These log-log drawings show the graphical comparison of the two maximal Prime Gaps Lower Bounds: Ford et all $\mathcal{P} \mathcal{B}$ (Blue) and the $\mathcal{L B}$ (Red). The R.H. drawing shows the difference $\mathcal{L B}-\mathcal{P B}$. Both figures are drawn over the range $p_{(n+1)} \in \mathbb{N} \mid 4652507 \leq p_{(n+1)} \leq 18361375334787048247$.

The lower bound on Maximal Prime Gaps, presented by Ford et al [16], attains values that are less than zero over the range of the maximal prime gaps for $p_{(n+1)} \in \mathbb{N} \mid 11 \leq p_{(n+1)} \leq 2010881$. Please refer to Table 5 in the Appendix. Consequently, the log-log figures can only be drawn for $p_{(n+1)} \in \mathbb{N} \mid p_{(n+1)} \geq 4652507$. Pragmatically, we prove Theorem 3.5, beginning at the lowest $\mathcal{C}$ value. This means, at the prime gap terminating at $p_{(n+1)}=19581334193189$. The value of the constant $\mathcal{C}$ is only an approximation, as it is computed w.r.t. the Lower Bound, given by Theorem 3.4. The approximation is however sufficient and suitable, to compute the approximate value of $\mathcal{C}$, at any technically feasible (or desired) point $n \in \mathbb{N} \mid n \geq 4652507$.

Corollary 3.5 (Maximal Prime Gaps Lower Bound $\mathcal{C}$ Value).

The primary lower bound for all $p_{(n+1)} \in \mathbb{N} \mid p_{(n+1)} \geq 4652507$ (Ford et al [16]), is given by:

$$
\mathfrak{g}=p_{(n+1)}-p_{(n)} \gg \frac{(\log N)(\log \log N)(\log \log \log \log N)}{\log \log \log N}=\mathcal{P B}
$$

The implicit constant $\mathcal{C}$ can be estimated by implementing $\mathcal{C} \gtrsim \mathcal{L} \mathcal{B}_{p_{(n+1)}} / \mathcal{P} \mathcal{B}_{p_{(n+1)}}$. The value of $\mathcal{C}$, for $p_{(n+1)} \geq 4652507$, is $\mathcal{C} \geq 32$, and gradually increases unboundedly as $p_{(n+1)}$ tends to infinity.

Remark 3.1. Corollary 3.5 is a direct consequence of Theorem 3.4. Hence, a simple validation is sufficient.

Proof.

By Theorems 1.1 and 3.4 we have:

$$
\left(\mathcal{M} \times\left(5\left(\log _{10} p_{n}\right)^{\alpha}-7\left(\log _{10} p_{n}\right)^{(2 \gamma)}\right)+\beta\right) \leq\left(p_{n+1}-p_{n}\right) \leq 5\left(\log _{10} p_{n}\right)^{2}
$$

Kevin Ford Et Al. [16] have shown, that for any sufficiently large $p_{(n+1)} \leq N$ :

$$
\mathfrak{g}=p_{(n+1)}-p_{(n)} \gg \frac{\left(\log p_{(n+1)}\right)\left(\log \log p_{(n+1)}\right)\left(\log \log \log \log p_{(n+1)}\right)}{\log \log \log p_{(n+1)}}=\mathcal{P B}
$$


The difference:

$$
\left(\mathcal{L} \mathcal{B}_{p_{(n+1)}}-\mathcal{P} \mathcal{B}_{p_{(n+1)}}\right)
$$

At $p_{(n+1)}=19581334193189$ attains 552.277785 and increases. Please refer to Figure 7 and Table 5. Implementing Cauchy Root Test:

$$
\sqrt[j]{\mathcal{C}_{j}}=\sqrt[j]{\left(\frac{\mathcal{L} \mathcal{B}_{p_{(n+1)}}}{\mathcal{P} \mathcal{B}_{p_{(n+1)}}}\right)}
$$

where $j \in \mathbb{N} \mid j \geq 55$ is the ordinal number of the maximal prime gap. At $p_{(n+1)}=19581334193189$ the Cauchy Root Test attains approx. 1.06525 and converges asymptotically, strictly from above to 1 . Please refer to Table 5 . Consequently, by the definition of the Cauchy Root Test, the ratio $\mathcal{C}_{j}$ diverges for $p_{(n+1)} \geq$ 19581334193189. This implies that the difference 3.18 also diverges. The ratio $\mathcal{C}_{j}=\left(\mathcal{L B}_{p_{(n+1)}}\right) /\left(\mathcal{P} \mathcal{B}_{p_{(n+1)}}\right)$ at $p_{(n+1)}=19581334193189$, attains 32.3403523719 and further increases at every step $j$, as $p_{(n+1)}$ increases unboundedly. Please refer to Figure 8. This shows that Corollary 3.5 is valid for $p_{(n+1)} \geq 19581334193189$. For $4652507 \leq p_{(n+1)} \leq 19581334193189$, Figure 8 shows that Corollary 3.5 is true in that range too. Thus, completing the validation.
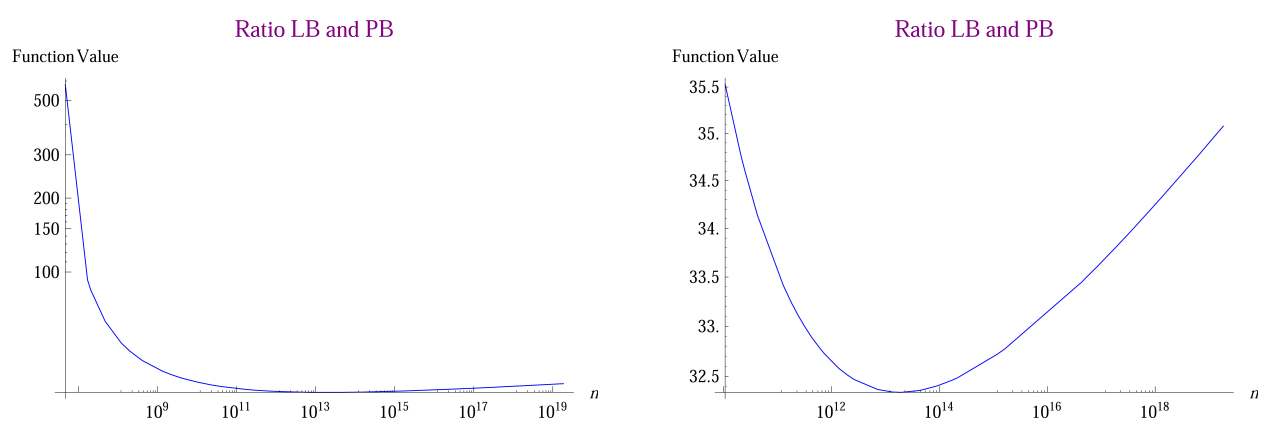

FiguRE 8. These log-log drawings show the ratio $\frac{\mathcal{L B}}{\mathcal{P B}}$.

The L.H. figure is drawn over the range:

$p_{(n+1)} \in \mathbb{N} \mid 4652507 \leq p_{(n+1)} \leq 18361375334787048247$.

The R.H. figure is drawn over the range:

$p_{(n+1)} \in \mathbb{N} \mid 10726905041 \leq p_{(n+1)} \leq 18361375334787048247$.

The global minimum occurs at $p_{(n+1)}=19581334193189$ where

the ratio attains approx. 32.34035237 .

The graphs in the Figure 9 have been generated to simply assist in visualization, of the $\mathcal{C}$ constant's slow rate of divergence and/or convergence of the Cauchy Root Test. Although the figures are not a part of the verification itself, they aid to understand the concept and help to shape up our expectations. 

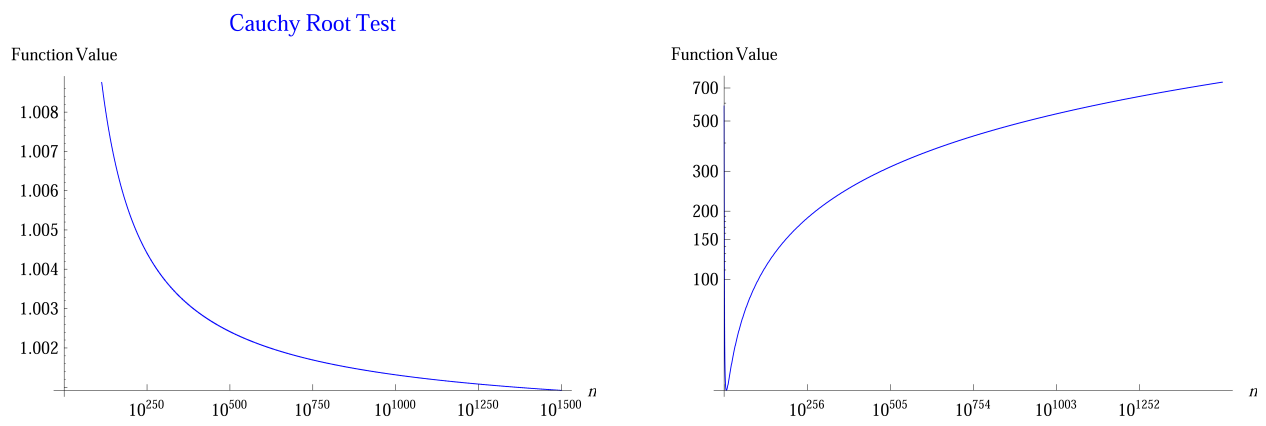

Figure 9. The L.H. drawing shows the Cauchy Root Test of the ratio $\frac{\mathcal{L} \mathcal{B}}{\mathcal{P B}}$. The R.H. drawing shows the ratio $\frac{\mathcal{L B}}{\mathcal{P B}}$. Both figures are drawn in the range $n \in \mathbb{N} \mid 4652507 \leq \approx 7.662607 \times 10^{1499}$.

\section{REFERENCES}

[1] R.J. Backlund, Über die Differenzen zwischen den Zahlen, die zu den ersten $n$ Primzahlen teilerfremd sind. Commentationes in honorem Ernesti Leonardi Lindelöf, Annales Acad. Sci. Fenn. 32 (1929), no. 2, 1-9.

[2] Enrico Bombieri, The shifting aspects of truth in mathematics, Institute For Advanced Study Princeton (2008).

[3] A. Brauer and H. Zeitz, Über eine zahlentheoretische Behauptung von Legendre, Sitzungsbericht, Berliner Math. Ges. 29 (1930), 116-125.

[4] Viggo Brun, Über das Goldbachsche Gesetz und die Anzahl der Primzahlpaare, Arch. Mat. Natur (1915).

[5] Chris K. Caldwell, The gaps between primes (2006). http://primes.utm.edu/notes/gaps.html.

[6] Calvin C. Clawson, Mathematical mysteries; the beauty and magic of numbers, Plenum Press, New York, 1996.

[7] Harald Cramer, On the order of magnitude of the difference between consecutive prime numbers, Acta Arithmetica (1936).

[8] R. Crandall and C. Pomerance, Prime numbers, a computational perspective, Springer Verlag, New York, 2005.

[9] P. Dusart, Autour de la fonction qui compte le nombre de nombres premiers, Limoges, 1998.

[10] Paul Erdös, On the difference of consecutive primes, Quarterly Journal Of Mathematics (1935).

[11] _ Some unsolved problems, Publications Of The Mathematical Institute Of The Hungarian Academy Of Sciences (1961).

[12] _ A survey of problems in combinatorial number theory, Annals Of Discrete Mathematics (1980).

[13] Paul Erdös and E.G. Strauss, Remarks on the differences between consecutive primes, Elem. Math. 35 (1980), 115-118.

[14] Jan Feliksiak, The elementary proof of the Riemann's Hypothesis, MDPI, AG, https://doi.org/10.20944/preprints202006.0365.v1 (2020).

[15] _ The maximal prime gaps Supremum and the Firoozbakht's Hypothesis No 30, MDPI, AG, https://doi.org/10.20944/preprints202006.0366.v1 (2020).

[16] Kevin Ford, Ben Green, Sergei Konyagin, James Maynard, and Terence Tao, Long gaps between primes, arXiv:1412.5029v3[math.NT] (2016).

[17] D.R. Heath-Brown, Differences between consecutive primes, Jahresber. Deutsch. Mathem. Ver. 90 (1988), 71-89.

[18] Kenneth Ireland and Michael Rosen, A classical introduction to modern number theory, Springer Verlag N.Y., 1990.

[19] Helge von Koch, Sur la distribution des nombres premiers, Acta Mathematica (1901).

[20] Edmund Landau, Vorlesungen über Zahlentheorie, Vol. II, S. Hirzel, Leipzig, 1927.

[21] H Maier, Primes in short intervals, Michigan Mathematical Journal (1985). 
[22] Thomas R. Nicely, Some results of computational research in prime numbers (Computational number theory) (2009). http://www.trnicely.net.

[23] János Pintz, Very large gaps between consecutive primes, Journal Of Number Theory (1997).

[24] Robert A. Rankin, The difference between consecutive prime numbers, Journal London Mathematical Society (1938).

[25] H. Riesel, Prime numbers and computer methods for factorization, Birkháuser, Boston, 1994.

[26] Sebastian M. Ruiz, A result on prime numbers, Mathematical Gazette 81 (1997), no. 269, 269.

[27] Atle Selberg, On the normal density of primes in small intervals and the difference between consecutive primes, Arch. Mathem. B 47 (1943), 87-105.

[28] _ An elementary proof of the prime number theorem, Ann. Math. 50 (1949), 305-313.

[29] Daniel Shanks, On maximal gaps between successive primes, Math. Comp. 18 (1964), 646651.

[30] Tomas Oliveira e Silva, Gaps between consecutive primes (2006). www.ieeta.pt/ tos/gaps.html.

[31] E. Westzynthius, Über die Differenzen Verteilung der Zahlen die zu den $n$ ersten Primzahlen teilerfremd sind, Comm. Phys. Math. Soc. Sci. Fenn. 5 (1931), 1-37. 


\section{Appendix}

\subsection{Tabular data.}

TABle 1

\begin{tabular}{|c|c|c|c|}
\hline \multicolumn{4}{|c|}{ Maximal Prime Gaps Upper Bound } \\
\hline$N^{o}$ & Gap start $p_{n}$ & Actual gap $\mathfrak{g}$ & $\mathcal{U} \mathcal{B}_{\left(p_{n}\right)}$ \\
\hline 1 & 7 & 4 & 3 \\
\hline 2 & 23 & 6 & 9 \\
\hline 3 & 89 & 8 & 19 \\
\hline 4 & 113 & 14 & 21 \\
\hline 5 & 523 & 18 & 36 \\
\hline 6 & 887 & 20 & 43 \\
\hline 7 & 1129 & 22 & 46 \\
\hline 8 & 1327 & 34 & 48 \\
\hline 9 & 9551 & 36 & 79 \\
\hline 10 & 15683 & 44 & 88 \\
\hline 11 & 19609 & 52 & 92 \\
\hline 12 & 31397 & 72 & 101 \\
\hline 13 & 155921 & 86 & 134 \\
\hline 14 & 360653 & 96 & 154 \\
\hline 15 & 370261 & 112 & 155 \\
\hline 16 & 492113 & 114 & 161 \\
\hline 17 & 1349533 & 118 & 187 \\
\hline 18 & 1357201 & 132 & 188 \\
\hline 19 & 2010733 & 148 & 198 \\
\hline 20 & 4652353 & 154 & 222 \\
\hline 21 & 17051707 & 180 & 261 \\
\hline 22 & 20831323 & 210 & 267 \\
\hline 23 & 47326693 & 220 & 294 \\
\hline 24 & 122164747 & 222 & 326 \\
\hline 25 & 189695659 & 234 & 342 \\
\hline 26 & 191912783 & 248 & 343 \\
\hline 27 & 387096133 & 250 & 368 \\
\hline 28 & 436273009 & 282 & 373 \\
\hline 29 & 1294268491 & 288 & 415 \\
\hline 30 & 1453168141 & 292 & 419 \\
\hline 31 & 2300942549 & 320 & 438 \\
\hline 32 & 3842610773 & 336 & 459 \\
\hline 33 & 4302407359 & 354 & 464 \\
\hline 34 & 10726904659 & 382 & 503 \\
\hline 35 & 20678048297 & 384 & 532 \\
\hline 36 & 22367084959 & 394 & 535 \\
\hline 37 & 25056082087 & 456 & 540 \\
\hline 38 & 42652618343 & 464 & 564 \\
\hline 39 & 127976334671 & 468 & 616 \\
\hline
\end{tabular}


Table 1. Continued

\begin{tabular}{|l|l|l|l|}
\hline \multicolumn{5}{|l|}{ Maximal Prime Gaps Upper Bound (Continued) } \\
\hline 40 & 182226896239 & 474 & 634 \\
\hline 41 & 241160624143 & 486 & 647 \\
\hline 42 & 297501075799 & 490 & 658 \\
\hline 43 & 303371455241 & 500 & 659 \\
\hline 44 & 304599508537 & 514 & 659 \\
\hline 45 & 416608695821 & 516 & 675 \\
\hline 46 & 461690510011 & 532 & 680 \\
\hline 47 & 614487453523 & 534 & 694 \\
\hline 48 & 738832927927 & 540 & 704 \\
\hline 49 & 1346294310749 & 582 & 735 \\
\hline 50 & 1408695493609 & 588 & 737 \\
\hline 51 & 1968188556461 & 602 & 755 \\
\hline 52 & 2614941710599 & 652 & 770 \\
\hline 53 & 7177162611713 & 674 & 826 \\
\hline 54 & 13829048559701 & 716 & 863 \\
\hline 55 & 19581334192423 & 766 & 883 \\
\hline 56 & 42842283925351 & 778 & 929 \\
\hline 57 & 90874329411493 & 804 & 974 \\
\hline 58 & 171231342420521 & 806 & 1012 \\
\hline 59 & 218209405436543 & 906 & 1028 \\
\hline 60 & 1189459969825483 & 916 & 1136 \\
\hline 61 & 1686994940955803 & 924 & 1159 \\
\hline 62 & 1693182318746371 & 1132 & 1159 \\
\hline 63 & 43841547845541059 & 1184 & 1384 \\
\hline 64 & 55350776431903243 & 1198 & 1401 \\
\hline 65 & 80873624627234849 & 1220 & 1429 \\
\hline 66 & 203986478517455989 & 1224 & 1498 \\
\hline 67 & 218034721194214273 & 1248 & 1503 \\
\hline 68 & 305405826521087869 & 1272 & 1528 \\
\hline 69 & 352521223451364323 & 1328 & 1539 \\
\hline 70 & 401429925999153707 & 1356 & 1549 \\
\hline 71 & 418032645936712127 & 1370 & 1552 \\
\hline 72 & 804212830686677669 & 1442 & 1603 \\
\hline 73 & 1425172824437699411 & 1476 & 1647 \\
\hline 74 & 5733241593241196731 & 1488 & 1759 \\
\hline 75 & 6787988999657777797 & 1510 & 1773 \\
\hline 76 & 15570628755536096243 & 1526 & 1841 \\
\hline 77 & 17678654157568189057 & 1530 & 1852 \\
\hline 78 & 18361375334787046697 & 1550 & 1855 \\
\hline & & The End & \\
\hline & & & \\
\hline
\end{tabular}


TABle 2

\begin{tabular}{|l|l|l|l|l|}
\hline \multicolumn{5}{|c|}{ Infimum on the Maximal Prime Gaps } \\
\hline$N^{\circ}$ & Gap start $p_{n}$ & Actual gap $\mathfrak{g}$ & Infimum & Est. Error \\
\hline 1 & 7 & 4 & - & - \\
\hline 2 & 23 & 6 & 5.78012 & 0.21988 \\
\hline 3 & 89 & 8 & 7.922911 & 0.077089 \\
\hline 4 & 113 & 14 & 13.826016 & 0.173984 \\
\hline 5 & 523 & 18 & 17.950855 & 0.049145 \\
\hline 6 & 887 & 20 & 19.966698 & 0.033302 \\
\hline 7 & 1129 & 22 & 21.969323 & 0.030677 \\
\hline 8 & 1327 & 34 & 33.939380 & 0.06062 \\
\hline 9 & 9551 & 36 & 35.991954 & 0.008046 \\
\hline 10 & 15683 & 44 & 43.992819 & 0.007181 \\
\hline 11 & 19609 & 52 & 51.992085 & 0.007915 \\
\hline 12 & 31397 & 72 & 71.99072 & 0.00928 \\
\hline 13 & 155921 & 86 & 85.996433 & 0.003567 \\
\hline 14 & 360653 & 96 & 95.997221 & 0.002779 \\
\hline 15 & 370261 & 112 & 111.996601 & 0.003399 \\
\hline 16 & 492113 & 114 & 113.996872 & 0.003128 \\
\hline 17 & 1349533 & 118 & 117.997421 & 0.002579 \\
\hline 18 & 1357201 & 132 & 131.997069 & 0.002931 \\
\hline 19 & 2010733 & 148 & 147.996839 & 0.003161 \\
\hline 20 & 4652353 & 154 & 153.996917 & 0.003083 \\
\hline 21 & 17051707 & 180 & 179.9965 & 0.0035 \\
\hline 22 & 20831323 & 210 & 209.995915 & 0.004085 \\
\hline 23 & 47326693 & 220 & 219.995734 & 0.004266 \\
\hline 24 & 122164747 & 222 & 221.995687 & 0.004313 \\
\hline 25 & 189695659 & 234 & 233.995446 & 0.004554 \\
\hline 26 & 191912783 & 248 & 247.995172 & 0.004828 \\
\hline 27 & 387096133 & 250 & 249.995118 & 0.004882 \\
\hline 28 & 436273009 & 282 & 281.994489 & 0.005511 \\
\hline 29 & 1294268491 & 288 & 287.99434 & 0.00566 \\
\hline 30 & 1453168141 & 292 & 291.994258 & 0.005742 \\
\hline 31 & 2300942549 & 320 & 319.993692 & 0.006308 \\
\hline 32 & 3842610773 & 336 & 335.993359 & 0.006641 \\
\hline 33 & 4302407359 & 354 & 353.9929995 & 0.0070005 \\
\hline 34 & 10726904659 & 382 & 381.992411 & 0.007589 \\
\hline 35 & 20678048297 & 384 & 383.992347 & 0.007653 \\
\hline 36 & 22367084959 & 394 & 393.992145 & 0.007855 \\
\hline 37 & 25056082087 & 456 & 455.990905 & 0.009095 \\
\hline 38 & 42652618343 & 464 & 463.990722 & 0.009278 \\
\hline 39 & 127976334671 & 467.990596 & 0.009404 \\
\hline 40 & 182226896239 & 474.99046 & 0.00954 \\
\hline 41 & 241160624143 & 485.990207 & 0.009793 \\
\hline 42 & 297501075799 & 489.990118 & 0.009882 \\
\hline & & & & \\
\hline
\end{tabular}


TABle 2. Continued

\begin{tabular}{|l|l|l|l|l|}
\hline \multicolumn{5}{|c|}{ Infimum (Continued) } \\
\hline 43 & 303371455241 & 500 & 499.989915 & 0.010085 \\
\hline 44 & 304599508537 & 514 & 513.989632 & 0.010368 \\
\hline 45 & 416608695821 & 516 & 515.989578 & 0.010422 \\
\hline 46 & 461690510011 & 532 & 531.989251 & 0.010749 \\
\hline 47 & 614487453523 & 534 & 533.989198 & 0.010802 \\
\hline 48 & 738832927927 & 540 & 539.989067 & 0.010933 \\
\hline 49 & 1346294310749 & 582 & 581.988189 & 0.011811 \\
\hline 50 & 1408695493609 & 588 & 587.988065 & 0.011935 \\
\hline 51 & 1968188556461 & 602 & 601.987765 & 0.012235 \\
\hline 52 & 2614941710599 & 652 & 651.986733 & 0.013267 \\
\hline 53 & 7177162611713 & 674 & 673.986233 & 0.013767 \\
\hline 54 & 13829048559701 & 716 & 715.98534 & 0.01466 \\
\hline 55 & 19581334192423 & 766 & 765.984297 & 0.015703 \\
\hline 56 & 42842283925351 & 778 & 777.984006 & 0.015994 \\
\hline 57 & 90874329411493 & 804 & 803.983429 & 0.016571 \\
\hline 58 & 171231342420521 & 806 & 805.983352 & 0.016648 \\
\hline 59 & 218209405436543 & 906 & 905.981272 & 0.018728 \\
\hline 60 & 1189459969825483 & 916 & 915.98096 & 0.01904 \\
\hline 61 & 1686994940955803 & 924 & 923.980774 & 0.019226 \\
\hline 62 & 1693182318746371 & 1132 & 1131.976444 & 0.023556 \\
\hline 63 & 43841547845541059 & 1184 & 1183.975126 & 0.024874 \\
\hline 64 & 55350776431903243 & 1198 & 1197.974816 & 0.025184 \\
\hline 65 & 80873624627234849 & 1220 & 1219.974325 & 0.025675 \\
\hline 66 & 203986478517455989 & 1224 & 1223.974178 & 0.025822 \\
\hline 67 & 218034721194214273 & 1248 & 1247.973664 & 0.026336 \\
\hline 68 & 305405826521087869 & 1272 & 1271.973136 & 0.026864 \\
\hline 69 & 352521223451364323 & 1328 & 1327.971942 & 0.028058 \\
\hline 70 & 401429925999153707 & 1356 & 1355.97134 & 0.02866 \\
\hline 71 & 418032645936712127 & 1370 & 1369.971039 & 0.028961 \\
\hline 72 & 804212830686677669 & 1442 & 1441.969466 & 0.030534 \\
\hline 73 & 1425172824437699411 & 1476 & 1475.9687 & 0.0313 \\
\hline 74 & 5733241593241196731 & 1488 & 1487.968334 & 0.031666 \\
\hline 75 & 6787988999657777797 & 1510 & 1509.967856 & 0.032144 \\
\hline 76 & 15570628755536096243 & 1526 & 1525.967448 & 0.032552 \\
\hline 77 & 17678654157568189057 & 1530 & 1529.967353 & 0.032647 \\
\hline 78 & 18361375334787046697 & 1550 & 1549.966923 & 0.033077 \\
\hline & The End & & \\
\hline & & & & \\
\hline
\end{tabular}


TABle 3

\begin{tabular}{|l|l|l|l|l|}
\hline \multicolumn{5}{|c}{ Supremum on the Maximal Prime Gaps } \\
\hline$N^{\circ}$ & Gap start $p_{n}$ & Actual gap $\mathfrak{g}$ & Supremum & Est. Error \\
\hline 1 & 7 & 4 & - & - \\
\hline 2 & 23 & 6 & 5.780362 & -0.219638 \\
\hline 3 & 89 & 8 & 7.923243 & -0.076757 \\
\hline 4 & 113 & 14 & 13.826595 & -0.173405 \\
\hline 5 & 523 & 18 & 17.951607 & -0.048393 \\
\hline 6 & 887 & 20 & 19.967535 & -0.032465 \\
\hline 7 & 1129 & 22 & 21.970244 & -0.029756 \\
\hline 8 & 1327 & 34 & 33.940803 & -0.059197 \\
\hline 9 & 9551 & 36 & 35.993464 & -0.006536 \\
\hline 10 & 15683 & 44 & 43.994664 & -0.005336 \\
\hline 11 & 19609 & 52 & 51.994266 & -0.005734 \\
\hline 12 & 31397 & 72 & 71.993739 & -0.006261 \\
\hline 13 & 155921 & 86 & 86.00004 & 0.00004 \\
\hline 14 & 360653 & 96 & 96.001247 & 0.001247 \\
\hline 15 & 370261 & 112 & 112.001298 & 0.001298 \\
\hline 16 & 492113 & 114 & 114.001654 & 0.001654 \\
\hline 17 & 1349533 & 118 & 118.002371 & 0.002371 \\
\hline 18 & 1357201 & 132 & 132.002606 & 0.002606 \\
\hline 19 & 2010733 & 148 & 148.003048 & 0.003048 \\
\hline 20 & 4652353 & 154 & 154.003377 & 0.003377 \\
\hline 21 & 17051707 & 180 & 180.004052 & 0.004052 \\
\hline 22 & 20831323 & 210 & 210.004725 & 0.004725 \\
\hline 23 & 47326693 & 220 & 220.004964 & 0.004964 \\
\hline 24 & 122164747 & 222 & 222.005001 & 0.005001 \\
\hline 25 & 189695659 & 234 & 234.005263 & 0.005263 \\
\hline 26 & 191912783 & 248 & 248.005578 & 0.005578 \\
\hline 27 & 387096133 & 250 & 250.005607 & 0.005607 \\
\hline 28 & 436273009 & 282 & 282.006321 & 0.006321 \\
\hline 29 & 1294268491 & 288 & 288.006424 & 0.006424 \\
\hline 30 & 1453168141 & 292 & 292.006510 & 0.006510 \\
\hline 31 & 2300942549 & 320 & 320.007119 & 0.007119 \\
\hline 32 & 3842610773 & 336 & 336.007458 & 0.007458 \\
\hline 33 & 4302407359 & 354 & 354.007854 & 0.007854 \\
\hline 34 & 10726904659 & 382 & 382.008441 & 0.008441 \\
\hline 35 & 20678048297 & 384 & 384.008461 & 0.008461 \\
\hline 36 & 22367084959 & 394 & 394.008679 & 0.008679 \\
\hline 37 & 25056082087 & 456 & 456.01004 & 0.01004 \\
\hline 38 & 42652618343 & 464 & 464.010193 & 0.010193 \\
\hline 39 & 127976334671 & 468.010236 & 0.010236 \\
\hline 40 & 182226896239 & 474.010352 & 0.010352 \\
\hline 41 & 241160624143 & 486.010603 & 0.010603 \\
\hline 42 & 297501075799 & 490.010681 & 0.010681 \\
\hline & & & & \\
\hline
\end{tabular}


TABle 3. Continued

\begin{tabular}{|l|l|l|l|l|}
\hline \multicolumn{5}{|c|}{ Supremum (Continued) } \\
\hline 43 & 303371455241 & 500 & 500.010898 & 0.010898 \\
\hline 44 & 304599508537 & 514 & 514.011203 & 0.011203 \\
\hline 45 & 416608695821 & 516 & 516.011233 & 0.011233 \\
\hline 46 & 461690510011 & 532 & 532.011577 & 0.011577 \\
\hline 47 & 614487453523 & 534 & 534.011608 & 0.011608 \\
\hline 48 & 738832927927 & 540 & 540.011730 & 0.011730 \\
\hline 49 & 1346294310749 & 582 & 582.012614 & 0.012614 \\
\hline 50 & 1408695493609 & 588 & 588.012742 & 0.012742 \\
\hline 51 & 1968188556461 & 602 & 602.013029 & 0.013029 \\
\hline 52 & 2614941710599 & 652 & 652.014097 & 0.014097 \\
\hline 53 & 7177162611713 & 674 & 674.014521 & 0.014521 \\
\hline 54 & 13829048559701 & 716 & 716.015391 & 0.015391 \\
\hline 55 & 19581334192423 & 766 & 766.016446 & 0.016446 \\
\hline 56 & 42842283925351 & 778 & 778.016659 & 0.016659 \\
\hline 57 & 90874329411493 & 804 & 804.017175 & 0.017175 \\
\hline 58 & 171231342420521 & 806 & 806.017181 & 0.017181 \\
\hline 59 & 218209405436543 & 906 & 906.019298 & 0.019298 \\
\hline 60 & 1189459969825483 & 916 & 916.019409 & 0.019409 \\
\hline 61 & 1686994940955803 & 924 & 924.019559 & 0.019559 \\
\hline 62 & 1693182318746371 & 1132 & 1132.02396 & 0.02396 \\
\hline 63 & 43841547845541059 & 1184 & 1184.024828 & 0.024828 \\
\hline 64 & 55350776431903243 & 1198 & 1198.025106 & 0.025106 \\
\hline 65 & 80873624627234849 & 1220 & 1220.025540 & 0.025540 \\
\hline 66 & 203986478517455989 & 1224 & 1224.025558 & 0.025558 \\
\hline 67 & 218034721194214273 & 1248 & 1248.026056 & 0.026056 \\
\hline 68 & 305405826521087869 & 1272 & 1272.026532 & 0.026532 \\
\hline 69 & 352521223451364323 & 1328 & 1328.02769 & 0.02769 \\
\hline 70 & 401429925999153707 & 1356 & 1356.028262 & 0.028262 \\
\hline 71 & 418032645936712127 & 1370 & 1370.028553 & 0.028553 \\
\hline 72 & 804212830686677669 & 1442 & 1442.030 & 0.030 \\
\hline 73 & 1425172824437699411 & 1476 & 1476.030664 & 0.030664 \\
\hline 74 & 5733241593241196731 & 1488 & 1488.030804 & 0.030804 \\
\hline 75 & 6787988999657777797 & 1510 & 1510.031245 & 0.031245 \\
\hline 76 & 15570628755536096243 & 1526 & 1526.031511 & 0.031511 \\
\hline 77 & 17678654157568189057 & 1530 & 1530.031588 & 0.031588 \\
\hline 78 & 18361375334787046697 & 1550 & 1550.031998 & 0.031998 \\
\hline & & The End & & \\
\hline & & & & \\
\hline
\end{tabular}


TABle 4

\begin{tabular}{|c|c|c|c|}
\hline \multicolumn{4}{|c|}{ Maximal Prime Gaps Lower Bound } \\
\hline$N^{o}$ & Gap start $p_{n}$ & Actual gap $\mathfrak{g}$ & $\mathcal{L B}_{\left(p_{n}\right)}$ \\
\hline 1 & 7 & 4 & - \\
\hline 2 & 23 & 6 & 4.187953 \\
\hline 3 & 89 & 8 & 5.42782 \\
\hline 4 & 113 & 14 & 5.929179 \\
\hline 5 & 523 & 18 & 11.194365 \\
\hline 6 & 887 & 20 & 13.835209 \\
\hline 7 & 1129 & 22 & 15.182401 \\
\hline 8 & 1327 & 34 & 16.134312 \\
\hline 9 & 9551 & 36 & 30.970394 \\
\hline 10 & 15683 & 44 & 35.630568 \\
\hline 11 & 19609 & 52 & 37.852172 \\
\hline 12 & 31397 & 72 & 42.781476 \\
\hline 13 & 155921 & 86 & 62.087339 \\
\hline 14 & 360653 & 96 & 73.741040 \\
\hline 15 & 370261 & 112 & 74.123637 \\
\hline 16 & 492113 & 114 & 78.330546 \\
\hline 17 & 1349533 & 118 & 94.233928 \\
\hline 18 & 1357201 & 132 & 94.327589 \\
\hline 19 & 2010733 & 148 & 100.943659 \\
\hline 20 & 4652353 & 154 & 115.842369 \\
\hline 21 & 17051707 & 180 & 141.000844 \\
\hline 22 & 20831323 & 210 & 145.104297 \\
\hline 23 & 47326693 & 220 & 162.552277 \\
\hline 24 & 122164747 & 222 & 183.972161 \\
\hline 25 & 189695659 & 234 & 194.369152 \\
\hline 26 & 191912783 & 248 & 194.647620 \\
\hline 27 & 387096133 & 250 & 211.835859 \\
\hline 28 & 436273009 & 282 & 214.838993 \\
\hline 29 & 1294268491 & 288 & 243.124468 \\
\hline 30 & 1453168141 & 292 & 246.240438 \\
\hline 31 & 2300942549 & 320 & 258.803570 \\
\hline 32 & 3842610773 & 336 & 273.193759 \\
\hline 33 & 4302407359 & 354 & 276.417801 \\
\hline 34 & 10726904659 & 382 & 303.175118 \\
\hline 35 & 20678048297 & 384 & 323.162466 \\
\hline 36 & 22367084959 & 394 & 325.596419 \\
\hline 37 & 25056082087 & 456 & 329.131748 \\
\hline 38 & 42652618343 & 464 & 345.952387 \\
\hline 39 & 127976334671 & 468 & 382.020819 \\
\hline 40 & 182226896239 & 474 & 394.001476 \\
\hline 41 & 241160624143 & 486 & 403.631907 \\
\hline 42 & 297501075799 & 490 & 410.923740 \\
\hline
\end{tabular}


TABle 4. Continued

\begin{tabular}{|l|l|l|l|}
\hline \multicolumn{4}{|c|}{ Maximal Prime Gaps Lower Bound (Continued) } \\
\hline 43 & 303371455241 & 500 & 411.60569 \\
\hline 44 & 304599508537 & 514 & 411.746751 \\
\hline 45 & 416608695821 & 516 & 422.754414 \\
\hline 46 & 461690510011 & 532 & 426.397665 \\
\hline 47 & 614487453523 & 534 & 436.616941 \\
\hline 48 & 738832927927 & 540 & 443.268043 \\
\hline 49 & 1346294310749 & 582 & 465.271350 \\
\hline 50 & 1408695493609 & 588 & 466.954333 \\
\hline 51 & 1968188556461 & 602 & 479.471048 \\
\hline 52 & 2614941710599 & 652 & 490.233868 \\
\hline 53 & 7177162611713 & 674 & 529.440803 \\
\hline 54 & 13829048559701 & 716 & 555.711929 \\
\hline 55 & 19581334192423 & 766 & 569.899725 \\
\hline 56 & 42842283925351 & 778 & 602.487386 \\
\hline 57 & 90874329411493 & 804 & 634.630708 \\
\hline 58 & 171231342420521 & 806 & 662.354842 \\
\hline 59 & 218209405436543 & 906 & 673.119558 \\
\hline 60 & 1189459969825483 & 916 & 750.817572 \\
\hline 61 & 1686994940955803 & 924 & 767.350344 \\
\hline 62 & 1693182318746371 & 1132 & 767.524491 \\
\hline 63 & 43841547845541059 & 1184 & 930.030304 \\
\hline 64 & 55350776431903243 & 1198 & 942.263033 \\
\hline 65 & 80873624627234849 & 1220 & 962.330541 \\
\hline 66 & 203986478517455989 & 1224 & 1012.166929 \\
\hline 67 & 218034721194214273 & 1248 & 1015.802441 \\
\hline 68 & 305405826521087869 & 1272 & 1034.296059 \\
\hline 69 & 352521223451364323 & 1328 & 1042.219511 \\
\hline 70 & 401429925999153707 & 1356 & 1049.420519 \\
\hline 71 & 418032645936712127 & 1370 & 1051.671730 \\
\hline 72 & 804212830686677669 & 1442 & 1088.346960 \\
\hline 73 & 1425172824437699411 & 1476 & 1120.927172 \\
\hline 74 & 5733241593241196731 & 1488 & 1202.164233 \\
\hline 75 & 6787988999657777797 & 1510 & 1212.210242 \\
\hline 76 & 15570628755536096243 & 1526 & 1262.198192 \\
\hline 77 & 17678654157568189057 & 1530 & 1269.930815 \\
\hline 78 & 18361375334787046697 & 1550 & 1272.242921 \\
\hline & & & \\
\hline & & End & \\
\hline
\end{tabular}


TABle 5

\begin{tabular}{|l|l|l|l|l|}
\hline \multicolumn{5}{|c}{ Maximal Prime Gaps Lower Bound (Ford et all). } \\
\hline$N^{\circ}$ & Max. Prime Gap Endp. & $\mathcal{P B}_{\left(p_{(n+1)}\right)}$ & Diff: $\mathcal{L B}-\mathcal{P B}$ & Cauchy Root Test \\
\hline 1 & 11 & - & - & - \\
\hline 2 & 29 & -34.555205 & 38.764478 & - \\
\hline 3 & 97 & -14.436398 & 20.035106 & - \\
\hline 4 & 127 & -13.160648 & 19.366295 & - \\
\hline 5 & 541 & -9.404357 & 20.755163 & - \\
\hline 6 & 907 & -8.592431 & 22.548443 & - \\
\hline 7 & 1151 & -8.258842 & 23.552841 & - \\
\hline 8 & 1361 & -8.035861 & 24.322807 & - \\
\hline 9 & 9587 & -5.838985 & 36.843322 & - \\
\hline 10 & 15727 & -5.342327 & 41.000285 & - \\
\hline 11 & 19661 & -5.121875 & 43.000837 & - \\
\hline 12 & 31469 & -4.662480 & 47.468766 & - \\
\hline 13 & 156007 & -3.121775 & 65.216427 & - \\
\hline 14 & 360749 & -2.314771 & 76.059679 & - \\
\hline 15 & 370373 & -2.289334 & 76.417379 & - \\
\hline 16 & 492227 & -2.014078 & 80.348099 & - \\
\hline 17 & 1349651 & -1.029834 & 95.265207 & - \\
\hline 18 & 1357333 & -1.024255 & 95.353452 & - \\
\hline 19 & 2010881 & -0.636680 & 101.58160 & - \\
\hline 20 & 4652507 & 0.1990 & 115.643978 & 1.37484 \\
\hline 21 & 17051887 & 1.517748 & 139.483311 & 1.24084 \\
\hline 22 & 20831533 & 1.723817 & 143.380688 & 1.22323 \\
\hline 23 & 47326913 & 2.576437 & 159.975942 & 1.19746 \\
\hline 24 & 122164969 & 3.577814 & 180.394389 & 1.17841 \\
\hline 25 & 189695893 & 4.048367 & 190.320814 & 1.16749 \\
\hline 26 & 191913031 & 4.060843 & 190.586809 & 1.16049 \\
\hline 27 & 387096383 & 4.818926 & 207.016949 & 1.15041 \\
\hline 28 & 436273291 & 4.949078 & 209.889930 & 1.14416 \\
\hline 29 & 1294268779 & 6.144875 & 236.979598 & 1.13522 \\
\hline 30 & 1453168433 & 6.273519 & 239.966925 & 1.13013 \\
\hline 31 & 2300942869 & 6.786506 & 252.017068 & 1.12463 \\
\hline 32 & 3842611109 & 7.363520 & 265.830242 & 1.11955 \\
\hline 33 & 4302407713 & 7.491331 & 268.926473 & 1.11554 \\
\hline 34 & 10726905041 & 8.532880 & 294.642239 & 1.11072 \\
\hline 35 & 20678048681 & 9.290264 & 313.872203 & 1.10673 \\
\hline 36 & 22367085353 & 9.381377 & 316.215042 & 1.10354 \\
\hline 37 & 25056082543 & 9.513303 & 319.618446 & 1.10051 \\
\hline 38 & 42652618807 & 10.134455 & 335.817933 & 1.09736 \\
\hline 39 & 127976335139 & 11.432607 & 370.588212 & 1.09415 \\
\hline 40 & 182226896713 & 11.854422 & 382.147054 & 1.09154 \\
\hline 41 & 241160624629 & 12.190328 & 391.441579 & 1.08911 \\
\hline 42 & 297501076289 & 12.442850 & 398.48089 & 1.08683 \\
\hline & & Continued $\ldots$ & \\
\hline
\end{tabular}


TABle 5. Continued

\begin{tabular}{|l|l|l|l|l|}
\hline \multicolumn{5}{|c}{ Maximal Prime Gaps Bound Ford et all (Continued) } \\
\hline 43 & 303371455741 & 12.466388 & 399.139302 & 1.08472 \\
\hline 44 & 304599509051 & 12.471255 & 399.275495 & 1.08272 \\
\hline 45 & 416608696337 & 12.849336 & 409.905078 & 1.08073 \\
\hline 46 & 461690510543 & 12.973730 & 413.423935 & 1.07888 \\
\hline 47 & 614487454057 & 13.320741 & 423.2962 & 1.07708 \\
\hline 48 & 738832928467 & 13.545106 & 429.722937 & 1.07538 \\
\hline 49 & 1346294311331 & 14.279345 & 450.992005 & 1.07369 \\
\hline 50 & 1408695494197 & 14.335014 & 452.619319 & 1.07215 \\
\hline 51 & 1968188557063 & 14.746922 & 464.724126 & 1.07065 \\
\hline 52 & 2614941711251 & 15.09820 & 475.135668 & 1.06922 \\
\hline 53 & 7177162612387 & 16.356341 & 513.084462 & 1.06781 \\
\hline 54 & 13829048560417 & 17.181710 & 538.530219 & 1.0665 \\
\hline 55 & 19581334193189 & 17.621939 & 552.277785 & 1.06525 \\
\hline 56 & 42842283926129 & 18.619276 & 583.86811 & 1.06405 \\
\hline 57 & 90874329412297 & 19.585240 & 615.045467 & 1.06292 \\
\hline 58 & 171231342421327 & 20.405138 & 641.949704 & 1.06184 \\
\hline 59 & 218209405437449 & 20.720335 & 652.399224 & 1.06077 \\
\hline 60 & 1189459969826399 & 22.946864 & 727.870708 & 1.05986 \\
\hline 61 & 1686994940956727 & 23.410328 & 743.940015 & 1.05888 \\
\hline 62 & 1693182318747503 & 23.415192 & 744.109299 & 1.0579 \\
\hline 63 & 43841547845542243 & 27.803846 & 902.226458 & 1.0573 \\
\hline 64 & 55350776431904441 & 28.123132 & 914.139901 & 1.0564 \\
\hline 65 & 80873624627236069 & 28.643866 & 933.686676 & 1.05556 \\
\hline 66 & 203986478517457213 & 29.921297 & 982.245633 & 1.0548 \\
\hline 67 & 218034721194215521 & 30.013632 & 985.788809 & 1.05397 \\
\hline 68 & 305405826521089141 & 30.481598 & 1003.814461 & 1.0532 \\
\hline 69 & 352521223451365651 & 30.681217 & 1011.538294 & 1.05242 \\
\hline 70 & 401429925999155063 & 30.862185 & 1018.558334 & 1.05167 \\
\hline 71 & 418032645936713497 & 30.918672 & 1020.753057 & 1.05093 \\
\hline 72 & 804212830686679111 & 31.833185 & 1056.513775 & 1.05028 \\
\hline 73 & 1425172824437700887 & 32.636763 & 1088.290409 & 1.04964 \\
\hline 74 & 5733241593241198219 & 34.606352 & 1167.557881 & 1.04911 \\
\hline 75 & 6787988999657779307 & 34.846692 & 1177.363551 & 1.04846 \\
\hline 76 & 15570628755536097769 & 36.032573 & 1226.165619 & 1.0479 \\
\hline 77 & 17678654157568190587 & 36.214561 & 1233.716254 & 1.04728 \\
\hline 78 & 18361375334787048247 & 36.268902 & 1235.974019 & 1.04667 \\
\hline & The End & \\
\hline
\end{tabular}

